\title{
La gobernanza y la gestión integrada de los recursos hídricos: un desafío para las comunidades indígenas
}

\author{
Governance and integrated management of water resources: a challenge for \\ indigenous communities
}

\author{
Victor Cartuche1, ${ }^{1, *}$, David Cartuche ${ }^{2}$, Cesar Neira ${ }^{3}$ y Lourdes González ${ }^{4}$ \\ ${ }^{1}$ Centro de Investigaciones Tropicales del Ambiente y Biodiversidad (CITIAB), Universidad Nacional de Loja, Ecuador \\ 2 Carrera de Turismo, Universidad Nacional de Loja, Ecuador \\ ${ }^{3}$ Carrera de Administración de Empresas, Universidad Nacional de Loja, Ecuador \\ ${ }^{4}$ Centro de Investigaciones Ambientales y de Desarrollo Sostenible (CIADES), Ecuador \\ *Autor para correspondencia: victor.cartuche@unl.edu.ec
}

Fecha de recepción del manuscrito: 23/09/2021 Fecha de aceptación del manuscrito: 13/12/2021 Fecha de publicación: 24/12/2021

\begin{abstract}
Resumen-En Ecuador, los sistemas comunitarios para el manejo del agua, están plenamente reconocidos y apegados al marco jurídico, de ahí la importancia de fortalecer las organizaciones locales para la gestión del agua mediante la mejora de sus capacidades de organización y operación. Los 6.727 sistemas comunitarios proveen $24 \%$ de la población del Ecuador, demostrando que especialmente en zonas rurales y periféricas el problema no es la ausencia de agua, sino una crisis de gobernabilidad. Se llevó a cabo un estudio en la microcuenca del río Paquizhapa, sur de Ecuador, con el propósito de plantear estrategias de gestión integral del agua bajo un enfoque de gobernanza efectiva. Aplicamos técnicas de investigación bibliográfica, para recopilar y analizar experiencias exitosas de gestión del agua en América Latina. Se analizaron aspectos jurídicos y políticas vinculadas a la gestión social del agua. Fue necesaria una caracterización socioeconómica y ambiental de la microcuenca así como información relacionada con problemas de abastecimiento y cobertura de agua para consumo y regadío. Los resultados revelaron que, en la microcuenca las normativas para la administración del agua son débiles y carentes de criterios que generen corresponsabilidad entre los usuarios. La presencia de conflictos socio-ambientales, escasos impactos de inversiones de ONGs y organismos públicos, ausencia de liderazgo, débil articulación entre actores, genera una crisis en la gestión del agua siendo necesario impulsar estrategias basadas en la gestión por cuenca hidrográfica, las realidades locales y experiencias exitosas. Se plantea por lo tanto estrategias como: Gobernanza, ordenación del territorio, gestión de la demanda, gestión de ecosistemas acuáticos y gestión de la cooperación.
\end{abstract}

Palabras clave-Microcuenca, Gestión integral del agua, Gobernanza, Cuenca hidrográfica.

\begin{abstract}
In Ecuador, community water management systems are fully recognized and comply with the legal framework, hence the importance of strengthening local water management organizations by improving their organizational and operational capacities. The 6727 community systems provide water to $24 \%$ of the population, demonstrating that especially in rural and peripheral areas the problem is not the water absence, but a crisis of governance. A study was carried out in the micro-watershed of Paquizhapa river, southern Ecuador, with the purpose of proposing strategies for integrated water management under an effective governance approach. We applied bibliographic research techniques to compile and analyze successful water management experiences in Latin America. Legal aspects and policies related to social water management were analyzed. A socioeconomic and environmental characterization of the micro-watershed was required, as well as information related to problems of water supply and water coverage for consumption and irrigation. The results revealed that, although regulations exist in the micro-watershed, they are weak and lack criteria that generate responsibility among users. The presence of socio-environmental conflicts, scarce impacts of investments by ONGs and public agencies, lack of leadership, weak articulation between actors, generates a crisis in water management, making it necessary to promote strategies based on river basin management, local realities and successful experiences. Therefore, strategies such as: Governance, spatial planning, demand management, management of aquatic ecosystems and cooperation management were proposed.
\end{abstract}

Keywords-Microbasin, Integrated water management, Governance, Hydrographic basin. 


\section{INTRODUCCIÓN}

$\mathbf{E}$ 1 agua es un recurso natural imprescindible para el desarrollo social y económico de los pueblos. Este recurso estratégico, aunque domina la superficie de nuestro planeta sólo el $1 \%$ es agua dulce (Pardo, 2004), de manera que es un recurso sumamente escaso además que sus condiciones de calidad disminuyen drásticamente por la intervención del hombre. En la región de América Latina 77 millones de personas carecen de servicios de agua potable (Blanco et al., 2012) y entre una de las principales causas de mortalidad infantil se debe a problemas asociadas con la calidad del agua, (Alleyne, 2002) y se presentan más de 200 muertes diarias de niños menores a 5 años.

La gestión del agua en Sudamérica en la mayoría de casos se encuentra sectorizada y ciertos países ya la han privatizado como en caso de Chile a través de los mercados del agua (Larraín, 2006). En el Ecuador desde 2008, la gestión del agua se alinea a nuevas políticas y leyes que plantean mecanismos de gestión colectiva y, acuerdo a la (Constitución política del Ecuador, 2008), se reconoce el derecho de las comunidades, pueblos y nacionalidades ancestrales a usar, administrar y beneficiarse de los recursos naturales renovables existentes en sus tierras, siendo el agua uno de estos recursos; además promueve políticas sociales para la administración del agua reconociendo y garantizando a las organizaciones comunitarias de agua, comunas, comunidades, pueblos y nacionalidades indígenas. La misma constitución garantiza que la gestión del agua será sólo pública y comunitaria, con lo cual serán reconocidas y fortalecidas las iniciativas comunitarias en torno a la gestión del agua y la prestación de los servicios públicos.

La microcuenca del río Paquizhapa ubicada al sur del Ecuador dentro de la cuenca hidrográfica del río Jubones con una superficie de 12.258,77 hectáreas, abastece con agua para consumo y riego para una población directa de unos 7.150 habitantes e indirectamente a una población 138212 habitantes aproximadamente. Un análisis en cuanto a los aspectos biofísicos de la microcuenca determina que es un territorio mayormente cubierto por páramos, aunque también hay gran abundancia de pastos, bosques, cultivos de maíz, vegetación arbustiva y cultivos de ciclo corto. La microcuenca enfrenta importantes amenazas debido a las intervenciones humanas tales como la ganadería, agricultura, concesiones mineras y proyectos de vialidad lo que ponen en riesgo el estado de conservación especialmente de las zonas de recarga hídrica tales como páramo y bosque nativo factores que han disminuido la cantidad y calidad de agua.

Un diagnóstico socio-organizacional basado en un análisis DAFO y la determinación de la problemática indican que, la gestión del agua dentro de la microcuenca se lleva a cabo mediante sistemas de organización comunitaria a través de la conformación de Juntas o Comités de agua, las mismas que son encargadas del mantenimiento y manejo de los sistemas de agua. Este sistema de organización para la gestión del agua está reconocido dentro de la constitución ecuatoriana sin embargo, el estado no cumple su rol de apoyo para el fortalecimiento de las capacidades de estas organizaciones en términos del mejoramiento de su desempeño para una eficiente provisión de servicios de agua.

Además, es evidente la ausencia de mecanismos de coordinación entre instancias competentes presentándose por lo tanto una deficiente gobernanza de los recursos hídricos en el territorio. Tanto las intervenciones antrópicas como los deficientes mecanismos de gestión del agua en el territorio finalmente terminan afectando a las posibilidades de acceso al recurso hídrico en términos de calidad y en cantidades suficientes.

En este contexto, con esta investigación nos proponemos levantar una línea base en cuanto a las realidades en materia de gestión del agua en la microcuenca como herramienta para proponer medidas y estrategias que fortalezcan el desempeño de las Juntas o Comités de agua de las comunidades de cara a implementar una efectiva gobernanza de los recursos hídricos bajo criterios de gestión colectiva en el marco de los derechos planteados en la constitución. Nos planteamos, por lo tanto, definir estrategias vinculadas con: la gobernanza del agua, ordenación del territorio, la gestión de la demanda, la gestión de los ecosistemas acuáticos y la cooperación, todas propuestas de acuerdo a las realidades ambientales, socioorganizacionales de la microcuenca establecidas en la fase de diagnóstico, pero además bajo la transversalización de los postulados de la nueva cultura del agua y la gestión integrada de recursos hídricos.

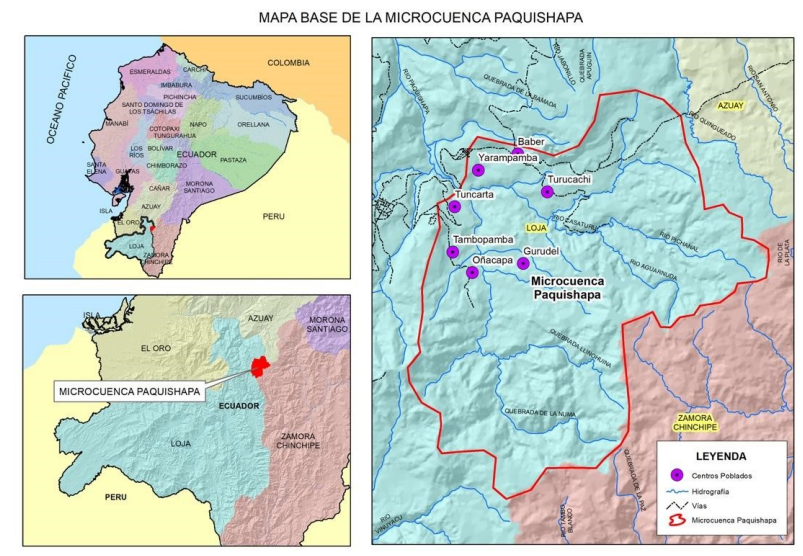

Fig. 1: Mapa de ubicación de la microcuenca Paquizhapa

La investigación abordo en una primera fase, la elaboración del estado del arte mediante la revisión bibliográfica e investigación sobre experiencias exitosas en gestión comunitaria del agua en territorios con similitudes sociales y naturales. Esta información permitió conocer estrategias, modelos implementados, resultados y experiencias alcanzadas que posibilitó reunir elementos como para determinar líneas estratégicas a implementarse en la microcuenca en estudio.

La siguiente fase, consistió en un diagnóstico a nivel de país relacionada a la gestión del agua. Situación análoga se efectuó para el cantón Saraguro. A nivel de microcuenca se realizó una caracterización de los aspectos socioorganizacionales, ambientales y económico-productivos vinculados con ciclo integral del agua. Este diagnóstico consistió en un análisis DAFO cuya información se obtuvo de fuentes bibliográficas secundarias, así como de entrevistas mantenidas con personas líderes de las comunidades. No se llevaron a cabo talleres insitu debido a que este trabajo fue realizado fuera del país.

La fase final consistió en la formulación de estrategias vinculadas con el manejo integral del agua en la microcuenca. Del estado del arte se obtuvo información sobre las meto- 
dologías, estrategias, modelos y mecanismos exitosos implementados en varias regiones. El planteamiento de estrategias abordó ejes orientados con la organización de los actores para una efectiva gobernanza del agua, la gestión de la demanda, ordenación del territorio, gestión de ecosistemas acuáticos y de la cooperación, los mismos que fueron trabajados bajo un enfoque de cuenca fluvial y de la nueva cultura del agua.

\section{RESULTADOS}

\section{Situación del agua en Ecuador}

Ecuador dispone de $43.500 \mathrm{~m}^{3}$ de agua por persona al año (2,5 veces superior al promedio mundial), sin embargo, es el país con más retraso en la región andina en cuanto a cobertura de servicios de agua y alcantarillado (tabla1), (Cabrera et al., 2012). Según (ENEMDU, 2016), el 76,51\% de los hogares a nivel nacional tiene acceso al servicio de agua potable, siendo el área urbana la que más acceso tiene con 9,7\% de los hogares (Tabla 1).

Más del $28 \%$ de la población ecuatoriana carece de acceso a agua por red pública y más del $22 \%$ no tiene una instalación de saneamiento adecuado (alcantarillado o pozo séptico) y alrededor de cuatro millones de personas se proveen de agua a través de pozos, ríos, vertientes, carros repartidores y agua lluvia (Córdova Guerrero, 2015).

En Ecuador solo 5 de cada 100 litros de aguas son tratadas antes de ser vertidos a los ríos y de los 218 Ayuntamientos que existen, solo 3 depuran su agua antes de verter a los ríos, (Córdova Guerrero, 2015).

Respecto a la gestión del agua y para efectos de mejorar su acceso, la inclusión y la participación social, el Ecuador en 2014 institucionalizó la Secretaría Nacional del Agua "SENAGUA" (Registro Oficial Suplemento 305, 2014), aunque desde 2021 pasó a ser una subsecretaría adscrita al Ministerio del Ambiente, Agua y Transición Ecológica. Esta Subsecretaría tiene el rol de liderar y conducir la gestión de los recursos hídricos de forma integrada y sustentable en los ámbitos de cuencas, subcuencas, microcuencas o demarcaciones hidrográficas e hidrogeológicas de acuerdo a la normativa vigente. En el contexto de los mecanismos de administración del agua, la Constitución política del Ecuador(2008), reconoce la forma pública y la comunitaria o la combinación de ambas como estrategias viables para le gestión del agua.

\section{La situación del agua en el cantón Saraguro}

El cantón de Saraguro se encuentra ubicado al norte de la provincia de Loja y su cabecera cantonal se halla a 2.525 msnm. Respecto al agua potable, según Senplades(2014), el acceso a agua entubada por red pública corresponde al $52,8 \%$; mientras que el porcentaje de viviendas sin alcantarillado en Saraguro es del $78 \%$, con lo cual se deduce que el saneamiento ambiental en el territorio es muy preocupante.

\section{La situación actual del agua en la microcuenca del río Paquizhapa}

La microcuenca del río Paquizhapa se ubica al sur del Ecuador, al norte de la provincia de Loja, en el cantón Saraguro, cubre una extensión de 12.258,77 hectáreas. Hidrográficamente se encuentra ubicada dentro de la cuenca del río Jubones, territorio que posee una superficie de 436.170 ha siendo la sexta cuenca más grande de la vertiente Occidental y la doceava a nivel nacional (Fajardo, 2010). Existe un clima frío durante casi todo el año, con temperaturas que oscilan entre los $12^{\circ} \mathrm{C}$ y $15^{\circ} \mathrm{C}$, durante el día, llegando en la noche hasta $\operatorname{los} 2^{\circ} \mathrm{C}$ a $3^{\circ} \mathrm{C}$. Predomina una topografía irregular de pendientes y medianas planicies.

Esta microcuenca abastece de agua para consumo y riego de manera directa a una población aproximada de 7.150 habitantes y de manera indirecta a una población de unas 138.212 personas aproximadamente (Cantones de la provincia del Oro) de la cuenca baja del Jubones. En cuanto a la cobertura vegetal, el mayor porcentaje de área se encuentra cubierto por páramos, aunque también hay gran abundancia de pastos, bosques y el resto de superficie cubierto por cultivos de maíz, vegetación arbustiva y cultivos de ciclo corto. La situación actual indica que en la microcuenca las intervenciones humanas (incendios, tala, pastoreo, agricultura), constituyen la amenaza principal para la destrucción de la cobertura vegetal provocando cambios en el uso del suelo. El páramo tiene una extensión de 3.401,94 ha, donde se encuentran humedales y sistemas lacustres, ecosistemas muy importantes en el almacenamiento y regulación hídrica. Sus vertientes de agua dan origen a la red hídrica de la cuenca del río Jubones y consecuentemente al aprovisionamiento de agua consumo humano para las poblaciones cuenca abajo.

\section{Acceso a agua y saneamiento}

Para este apartado, cabe la pena mencionar que, se carece de información oficial en torno a cifras y proyecciones y, en este contexto, lo que continuación se anota es producto del conocimiento personal adquirido por la experiencia al ser un miembro activo de una comunidad indígena de Saraguro. La situación actual indica que solo la comunidad Oñakapak cuenta con acceso al agua de tipo clorada demostrando que la cobertura de servicios de agua en las ciudades y cabeceras cantonales es superior a la cobertura de las áreas rurales. Ante esta situación las familias se abastecen de agua mediante una tubería desde las vertientes y zonas de recarga hídrica a través de tanques de captación. El agua llega a los surtidores de los domicilios donde debe ser hervida para su consumo, práctica que solo un porcentaje muy reducido de la población realiza.

En general, la infraestructura para la captación, conducción, almacenamiento y distribución del agua, se encuentra en malas condiciones y en casos esporádicos las comunidades organizan trabajos de mejoramiento y mantenimiento de los sistemas. En los sistemas comunitarios de agua el problema se presenta principalmente en las zonas de captación y recarga hídrica en donde se encuentran animales (pastoreo) alrededor de fuentes y captaciones de agua. Producto de aquello, los residuos orgánicos de los animales y por sedimentación son conducidos a los tanques de captación provocando contaminación orgánica, cambiando las características físicas y químicas del agua.

En cuanto al saneamiento, el $95 \%$ de la población carece de sistemas de alcantarillado y red de saneamiento. De este porcentaje únicamente un $90 \%$ de las familias cuentan con letrinas y pozos sépticos. Estas condiciones de insalubridad generan un problema serio de salud pública. Tan solo la co- 
Tabla 1: Cobertura de servicios de agua y alcantarillado por países (Cabrera et al., 2012)

\begin{tabular}{c|c|c|c|c|c|c}
\hline \multirow{2}{*}{ País } & \multicolumn{3}{|c|}{$\begin{array}{c}\text { Cobertura de } \\
\text { agua potable }\end{array}$} & \multicolumn{3}{c}{$\begin{array}{c}\text { Cobertura de } \\
\text { alcantarillado }\end{array}$} \\
\hline & Total & Urbana & Rural & Total & Urbana & Rural \\
\hline Bolivia & 73,5 & 93,1 & 44 & 63,5 & 82,3 & 35,3 \\
\hline Colombia & 90,6 & 98 & 73 & 83,4 & 97 & 51 \\
$>$ >- Ecuador & 70,3 & 81,5 & 51,4 & 58,0 & 70,5 & 37 \\
\hline Perú & 75,4 & 86,8 & 50,6 & 73,7 & 89,5 & 39,5 \\
\hline \multicolumn{7}{|c|}{}
\end{tabular}

munidad Tambopamba cuenta con un proyecto de alcantarillado mixto implementado en 2017. Sin embargo, sus aguas no tratadas son vertidas hacia el río Paquizhapa.

\section{La organización para la gestión del agua}

Cada una de las comunidades cuentan con un Comité de Agua, quienes llevan a cabo actividades de mantenimiento y operación de los sistemas para consumo y riego. Estas comisiones que la componen en promedio dos personas, en muy pocas ocasiones reciben un reconocimiento mensual o un salario por su servicio, como es el caso de Oñakapak y Tuncarta. Este aspecto genera que no se dediquen $100 \%$ a su trabajo encomendado con lo cual la administración del agua carece de un sistema de gestión responsable que asegure la calidad del servicio. Un aspecto importante es la baja participación de mujeres en espacios de dirección y gestión de los Comités de agua y en otras formas de organización comunitaria. En general cerca de un $80 \%$ de los puestos de dirección de los Gobiernos Comunitarios son asumidos por varones, perpetuando y consolidando la discriminación hacia la mujer.

En cuanto a las tarifas del agua, las comunidades: Tambopamba, Zhadan Pampa y Bahín Turukachi no establecen una tarifa por el consumo de agua, mientras que Oñakapak, Tuncarta, Gurudel y Baber si lo hacen. Este sistema de no pagar por el servicio de agua surge como un sistema arraigado a lo cultural, pues históricamente el servicio de agua ha sido catalogado como un patrimonio comunitario, donde los trabajos de mantenimiento y cuidado son totalmente de carácter comunitario bajo criterios de la solidaridad y reciprocidad. Sin embargo, en la actualidad se presentan problemas de desabastecimiento de agua, en gran medida generada por la ausencia de conciencia de ahorro, así como debido a las actividades antropogénicas que contaminan y destruyen cuerpos de agua y los efectos del cambio climático concretamente la presencia de periodos prolongados de sequía. Por ello varias de las comunidades han acordado establecer un pago de un valor monetario simbólico por el servicio de agua. De las siete comunidades, cuatro tienen establecido sistemas de cobro por consumo de agua (tabla 2). En algunos casos, las tarifas se pagan por consumo mensual $/ \mathrm{m}^{3}$ y en otros un pago mensual independientemente del consumo.

Un análisis de las debilidades, amenazas, fortalezas y oportunidades (DAFO) en la microcuenca reflejó que las principales fortalezas están relacionadas con la presencia de organizaciones de base como los cabildos y los Comités de agua que responden a un sistema ancestral de organización en donde prevalecen los criterios de solidaridad y reciprocidad para la administración del agua. Se destacan además aspectos como la voluntad y conciencia ambiental de la población.

Con respecto a las oportunidades se identificó a organis-
Tabla 2: Pago de tarifas por consumo de agua

\begin{tabular}{ccc}
\hline Comunidad & Tarifa/Tipo & Observaciones \\
\hline Oñakapak & $\$ 0,13 / \mathrm{m}^{3}$ & $\begin{array}{c}\text { Se encuentra en } \\
\text { organización } \\
\text { planificación. }\end{array}$ \\
\hline Tambopamba & No paga & $\begin{array}{c}\text { No se establece una } \\
\text { tarifa por } m^{3} . \\
\text { Solo es un } \\
\text { pago mensual. }\end{array}$ \\
\hline Shadanpamba & No paga & \\
\hline Bahín- & No paga & \\
\hline Gurukachi & $\$ 1 / \mathrm{mes}$ & $\begin{array}{c}\text { No se establece } \\
\text { una tarifa por } m^{3} .\end{array}$ \\
\hline Baber & $\$ 1 / \mathrm{mes}$ &
\end{tabular}

mos como el Ministerio del Ambiente, Agua y Transición Ecológica "MAATE", quien reconoce a los sistemas comunitarios como mecanismo válido de administración del agua, aspecto que garantiza el establecimiento de nuevos mecanismos comunitarios para la gestión, además de su fortalecimiento y la articulación de tareas con éstos en la prestación de servicios de agua potable y saneamiento, convirtiéndole en actor estratégico desde el punto de vista de la cooperación técnica y financiera. La microcuenca al estar ubicada dentro área de reserva de Biosfera Podocarpus el Cóndor (Serrano, 2008), adquiere un medio favorable para la gestión e implementación de proyectos de conservación de diversidad natural y cultural, donde agencias nacionales e internacionales han puesto un marcado interés. Además, el Ministerio del Ambiente y Transición Ecológica "MAATE" promueve políticas, proyectos y presupuestos para garantizar el estado de conservación de ecosistemas como bosque nativo y páramos. Igualmente, ONGs y Universidades podrían participar con inversiones e investigación, para monitorear el estado de conservación ecológica de ecosistemas acuáticos.

Entre las debilidades se identifican aspectos como la ausencia de liderazgo, capacidades locales y criterios de planificación limitados para la gestión del agua, carencia de criterios normativos que regulen y reglamenten el uso racional del agua, presencia de conflictos socioambientales, actividades antropogénicas que destruyen y contaminan fuentes de agua. Además, se evidencia un bajo nivel de participación y empoderamiento de los procesos de administración del agua por parte de los usuarios. La realidad es que la gran mayoría de la población carece de una cultura del ahorro, del cuidado y manejo responsable. La débil situación socio-organizativa ha generado problemas en cuanto a la gestión, articulación y coordinación con entidades públicas competentes, por lo 
que no se logra contar con recursos económicos y técnicos suficientes para satisfacer las necesidades de infraestructura hidráulica.

En cuanto a las amenazas, es una realidad que, las concesiones mineras en el territorio, preocupan a las comunidades y su área de influencia pues, los procesos que el Gobierno Central debía poner en marcha para promover la participación y el establecimiento de acuerdos con las comunidades han sido nulos. Si bien es cierto hasta la actualidad no se llevan a cabo procesos de extracción de minerales el hecho de solo existir concesiones mineras se convierte en una latente amenaza.

\section{Planteamiento de estrategias de gestión}

\section{Identificación de problemas principales y estrategias de intervención}

A raíz del análisis DAFO, se trabajó árbol de problemas (Tabla 3) bajo un enfoque de causa-efecto, que permitió identificar el principal problema dentro de la microcuenca, siendo este: la ausencia de un sistema de gestión sostenible del agua basada en estrategias que promuevan una efectiva gobernanza y gobernabilidad del agua.

En este escenario, se plantea una metodología de implementación de estrategias que integren a los actores del territorio bajo un enfoque de mancomunamiento, donde la diversidad de criterios permita tomar decisiones de forma colectiva (figura 2). Interesa, además, promover un manejo técnico adecuado de las áreas naturales de recarga hídrica. Adicionalmente, se plantean estrategias que incluyan medidas de ahorro y uso adecuado del agua. La cooperación efectiva entre actores comunitarios, públicos y privados en el marco de sus competencias y el desarrollo de programas de investigación científica y el diálogo de saberes, son también aspectos importantes planteados en las estrategias. Como eje transversal planteamos el diseño de un sistema de organización y gestión multinivel, donde instituciones de procedencia estatal y privada puedan articular sus objetivos a los ejes/estrategias planteadas. La meta es llegar a una efectiva coordinación y cogestión interinstitucional.

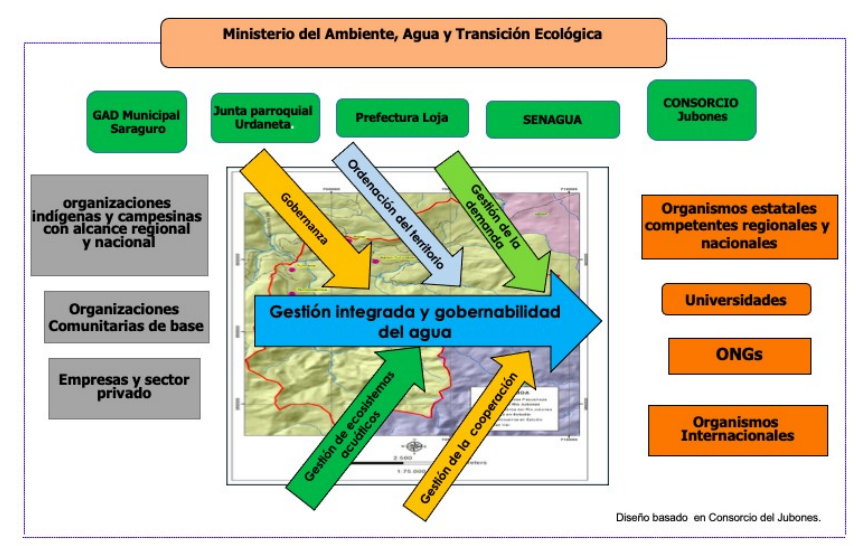

Fig. 2: Estrategias para la cogestión del agua

\section{Primera estrategia: gobernanza del agua}

Se basa en la participación de los actores sociales, públicos y privados dentro de la microcuenca, quienes podrían gene- rar capacidades de articulación buscando resolver problemas comunes. Por ello, la estrategia promueve una asociación voluntaria de organizaciones comunitarias, instituciones públicas competentes y organismos privados que trabajen en torno al agua para resolver problemas comunes y satisfacer sus necesidades territoriales dejando de lado limites geográficos. Se conformaría por lo tanto un Comité de Gestión con autonomía jurídica, roles y funciones específica en torno al agua y sus ámbitos de influencia directa. Este Comité de Gestión, se encargaría de promover la articulación con otras instancias públicas competentes como: El Municipio de Saraguro, la Junta Parroquial de Urdaneta, Gobierno Provincial de Loja, Universidades y el Ministerio del Ambiente, Agua y Transición Ecológica "MAATE".

\section{Segunda estrategia: ordenación del territorio}

Esta estrategia constituye un instrumento de carácter netamente técnico, con una proyección de planificación a mediano y largo plazo. La planificación para el uso adecuado del suelo con énfasis en los territorios de cabecera de cordilleras será uno de los ejes de trabajo más importantes para el Comité de Gestión. La formulación de las políticas, objetivos, así como la visión y misión serán construidas mediante la participación y concertación de actores sociales e institucionales de tal forma que el plan promueva la gestión del territorio de acuerdo con sus potencialidades y de manera especial la gestión de las áreas naturales de recarga hídrica.

\section{Tercera estrategia: gestión de la demanda}

La nueva cultura del agua, entre sus postulados propone un cambio que conduzca a que los procesos de gestión del agua se fundamenten en gestión de la demanda y de conservación de los ecosistemas acuáticos mediante el uso racional de los recursos hídricos (Agudo et al., 2009). En este ámbito, la estrategia propone un grupo de actuaciones que permitan la mejora de la gestión de los recursos hídricos disponibles, que ha de coordinarse estrechamente con los usos del suelo mediante los siguientes instrumentos:

1. Gestión técnica de los recursos hídricos, para optimizar los sistemas de regulación del agua de uso domiciliar y de regadío, para poder establecer un balance hídrico.

2. Estudios económicos para conocer los verdaderos costes de gestión del agua, desde su captación hasta el usuario final. La finalidad es construir mecanismos de asignación de agua basados en criterios de equilibrio, equidad y productividad.

3. Mecanismos que fomenten la participación de los usuarios y usuarias en la planificación y en la toma de decisiones, aspecto transversal en la gestión del agua basada en la demanda.

Con esta estrategia se pretende incluir un enfoque económico y técnico, como mecanismo que garantizar su gestión, uso y aprovechamiento de forma eficiente. Por lo tanto, la estrategia plantea medidas de ahorro y medidas de optimización, aterrizando finalmente en el criterio de "hacer más con menos", es decir menos uso del agua y mayor productividad. 


\section{Cuarta estrategia: gestión de ecosistemas acuáticos}

En la microcuenca, la crisis del agua y el desequilibrio en su ciclo natural se vinculan con las amenazas de las prácticas antropogénicas que destruyen los frágiles ecosistemas acuáticos. Estas amenazas tienen que ser disminuidas mediante propuestas direccionadas a la conservación de sus servicios ecosistémicos especialmente vinculados con la regulación y provisión de agua. El reto del sistema de organización dentro de la microcuenca es promover iniciativas para regular garantizar y generar programas para el uso eficiente del agua con responsabilidad ambiental con lo cual se podría garantizar el buen estado ecológico de estos ecosistemas acuáticos y con ello proveer agua de calidad y en cantidades suficientes. Por lo señalado, la estrategia que se propone se fundamenta en dos factores:

1. La consideración de que, en la microcuenca, los ríos, lagunas y páramos tienen un alto valor ecológico, puesto que almacenan una reserva importante de biodiversidad que son fundamentales en el mantenimiento del equilibrio ecológico (regulación hídrica y climática) y riqueza de fauna, flora y microorganismos.

2. La consideración de que estos ecosistemas acuáticos frágiles sufren actualmente una fuerte amenaza de destrucción y desaparición debido a presiones físicas, problemas ambientales globales y por razones socioeconómicas y culturales.

Al respecto de ejecutar cualquier acción de conservación y manejo integrado de los cuerpos de agua, la propuesta es llevar a cabo investigaciones de estos ecosistemas, que permitiría conocer información de su funcionalidad, su estado ecológico, etc., como insumos que permitan planificar para su manejo, conservación y restauración. Otro de los ejes importantes para perpetuar los objetivos de conservación es dedicar los esfuerzos para lograr la declaratoria de áreas protegidas a los territorios de importancia hídrica de la microcuenca.

Otro de los aspectos importantes que se debe considerar es el programa de educación ambiental que deberá propiciar la participación activa de diferentes públicos de la microcuenca mediante el uso de metodologías acordes a las realidades sociales, culturales y ambientales.

\section{Quinta estrategia: gestión para la cooperación}

La propuesta de cooperación interinstitucional y multinivel se constituirá en uno los ejes estratégicos para alcanzar la gestión integrada del agua y garantizar con ello su acceso adecuado para la población. De acuerdo a la información obtenida del DAFO, entre las oportunidades más destacadas se encuentra la presencia de organizaciones de cooperación especializadas en materia de agua y saneamiento, aspecto que abre la posibilidad para proponer mecanismos de trabajo coordinado.

Existen, además posibilidades para que la propuesta cuente con apoyo por parte de la cooperación internacional; por ello la idea es incluir a todas aquellas instancias que deseen ser parte de esta propuesta, siempre y cuando se vinculen a las políticas y a los mecanismos de cogestión que se plantea desde las comunidades. Será crucial, además, impulsar y participar activamente de la propuesta que el Municipio de
Saraguro actualmente promueve y lidera para la creación de áreas de conservación y uso sustentable "ACUS" una estrategia de conservación de carácter colectivo. En este contexto, hay que promover alianzas entre actores locales de la microcuenca que permitan articular herramientas comunes de gestión como es el caso de los planes de desarrollo y ordenamiento territorial Provinciales, cantonales y parroquiales como una estrategia que promueva la planificación y participación colectiva para la resolución de problemas comunes en torno al agua.

\section{El enfoque de género y la igualdad de oportunidades}

Será importante que todas las estrategias planteadas transverzalicen en enfoque de igualdad de género y oportunidades. Precisamente en la fase de diagnóstico, se identificó que hay diferencias en los derechos y deberes que se asignan a mujeres y hombres; aunque sabiendo que las mujeres están permanentemente presentes en diversas actividades económicas y sociales de las comunidades, sus aportes han sido invisibilizados.

En las comunidades que conforman la microcuenca, las mujeres son las que en la mayoría de casos se encargan del cuidado de la familia y el uso del agua para sustento familiar y en muchos casos para el regadío de cultivos, de manera que son ellas quienes conocen en profundidad las realidades en cuanto a la calidad y cantidad de agua y todo lo que aquello implica. Se propone que el Comité de Gestión de la microcuenca, deberán contar con un reglamento donde se estipule la participación de la mujer en igualdad de condiciones en cargos estratégicos y de responsabilidad compartida. Los objetivos, reglamentos y estatutos se direccionarán en fortalecer políticas acertadas para promover la igualdad entre los géneros y el empoderamiento de las mujeres en todos los procesos de gestión y decisión.

\section{DISCUSIÓN}

Los resultados obtenidos nos permiten demostrar que en la microcuenca Pakizhapa es posible implementar un modelo comunitario de gestión del agua basado en los postulados de la gestión integrada de recursos hídricos y la nueva cultura del agua, estrategias que permitirían tomar decisiones y gestionar el uso de los recursos hídricos superficiales desde el punto de vista de las necesidades bajo una perspectiva multidisciplinaria (Hantke, 2011) y además concebir al agua como un activo ecosocial (Aguilera, 2006) y que su gestión no es exclusivamente de carácter ingenieril si no de política social (Klink, 2018).

De acuerdo al diagnóstico, se presentan oportunidades y fortalezas principalmente vinculados con las competencias institucionales en materia de gestión del agua, un marco jurídico favorable que reconoce a los sistemas comunitarios de manejo del agua como mecanismos válidos para su gestión y además por los niveles importantes de organización de las comunidades para el manejo del agua, aspectos que posibilitan alcanzar una efectiva gobernanza y gobernabilidad del agua (Victoria, 2018).

Según los resultados, los sistemas comunitarios para la administración del agua con o sin reconocimiento jurídico, son organizaciones de base fundamentales para la provisión de 
agua en Latinoamérica (García Solis, 2011) de manera que la propuesta para la implementación de estrategias en la microcuenca está en completo apego a la constitución, misma que manifiesta claramente que la gestión del agua será exclusivamente de carácter público o comunitario, garantizando al mismo tiempo los medios para establecer alianzas de tipo público-comunitario de cara a proponer modelos de gestión sostenible del agua. Desde una perspectiva tanto desde el lado institucional en el marco de las competencias en materia de gestión del agua como de las dinámicas y fortalezas sociales que se presentan dentro de la microcuenca, es posible implementar actuaciones conjuntas para resolver problemas complejos en torno al acceso al agua con responsabilidad, democracia, eficiencia y transparencia, así, las Organizaciones Comunitarias de servicios de agua y saneamiento "OCSAS" son un ejemplo evidente de esta experiencia exitosa en la región latinoamericana debido a que alrededor de 80000 organizaciones a través de estatutos de autogobierno, trabajo mancomunado y elección de líderes de manera abierta, sencilla y democrática, dirigen sus esfuerzos a establecer un sistema de captación, potabilización, distribución y pago por el agua (Avina, 2011).

Experiencias positivas de gestión del agua a través de Comités de agua potable y saneamiento "CAPS", desarrolladas en Nicaragua (Umaña, 2016), eventualmente podrían extrapolarse a la propuesta de cogestión en la microcuenca $\mathrm{Pa}$ quizhapa, pues son modelos que se fundamentan en la participación activa de sus miembros para lograr acuerdos colectivos en cuanto a la administración del agua y servicios de saneamiento, destacando además estrategias como la capacitación a los Comités para generar conocimiento local en búsqueda de gestionar técnicamente no sólo los acueductos, sino el suelo y el bosque, construyendo obras sencillas de retención de escorrentías, muros de contención para proteger las áreas de influencia y afectaciones de las fuentes de agua, así como para evitar los desastres por inundación que afectan a las comunidades y sus bienes dentro de las microcuencas (Paz, 2013). Caso similar sucede con experiencias en Guatemala donde el éxito en la gestión comunitaria del agua se debe en al establecimiento y/o fortalecimiento de las relaciones de asociación con entidades gubernamentales, gobiernos locales, ONGs locales e internacionales que han brindado el soporte para complementar las actividades de las organizaciones comunitarias (Fundación Ipade, 2011).

En razón de la existencia de los medios y oportunidades para establecer acuerdos con organizaciones estatales que intervienen en la microcuenca Paquizhapa mediante acuerdos/convenios, sería viable la propuesta de una alianza público-comunitaria entre el Comité de Gestión, el Gobierno Local de Saraguro y el Gobierno Provincial de Loja, pues la constitución reconoce solo dos formas de gestión del agua: la pública y la comunitaria, de manera que un mecanismo de cogestión del agua sería a través de una alianza públicocomunitaria. Así lo refleja la experiencia exitosa del Centro de apoyo a la gestión rural del agua potable, CENAGRAP en Cañar, quienes desde 2002 trabajan a través de un modelo de alianza público-comunitaria en donde el Gobierno Municipal del cantón Cañar y 95 Juntas administradoras de agua potable (JAAPs) trabajan de manera articulada cuyo objetivo común es la sostenibilidad de éstas organizaciones y garantizar la dotación de agua en condiciones de calidad y en cantidades suficientes así como los servicios de saneamiento, (Medina, 2013).

\section{CONCLUSIONES}

Los resultados del análisis determinan que, en la microcuenca, a pesar de existir normativas comunitarias para la administración del agua, éstas carecen de criterios que generen responsabilidad en el manejo y gestión compartida entre los usuarios de agua, $\mathrm{y}$, si tenemos en cuenta realidades como la presencia de conflictos socio-ambientales, escasos impactos de inversiones de ONGs y organismos públicos, ausencia de liderazgo local, débil articulación entre actores, nos encontramos ante una realidad de crisis de gobernanza de los recursos hídricos.

Además, aunque la población demuestra un marcado interés para proteger y conservar los recursos hídricos, aún es necesario plantear, impulsar e implementar estrategias innovadoras para la gestión del agua que, por un lado tomen en cuenta las potencialidades locales en el contexto de su autodeterminación para la administración de los recursos naturales y, por otro lado, las oportunidades que la constitución determina en términos del reconocimiento legal a los Comités de agua de agua con atribuciones de administrar y dotar del servicio de agua. Adicionalmente, la presencia de Gobiernos Autónomos Descentralizados, instancias públicas como el MAATE y ONGs se constituyen en aliados estratégicos que, bajo instrumentos de cooperación, eventualmente serían parte de un Comité de Gestión de la microcuenca cuyo fin sería la implementación de un modelo de gestión que ponga en práctica las estrategias planteadas bajo la premisa de participación proactiva de los usuarios de agua y dirigentes de las comunidades.

\section{CONTRIBUCIONES DE LOS AUTORES}

Conceptualización: AC, DC y CN; metodología: AC; análisis formal: $\mathrm{AC}$; investigación: $\mathrm{AC}, \mathrm{DC}, \mathrm{LG}$ y CN; redacción - preparación del borrador original: AC, DC y CN; redacción - revisión y edición: AC y DC. Todos los autores han leído y aceptado la versión publicada del manuscrito. Alonso Cartuche: AC. David Cartuche: DC. Lourdes González: LG. Cesar Neira: $\mathrm{CN}$

\section{REFERENCIAS}

Agudo, P. A., Sánchez, V. P., y Colomina, G. B. (2009). Hacia una gestión sostenible del agua en Álava (Vol. 21). Fundación Nueva Cultura del Agua.

Aguilera, F. (2006). Hacia una nueva economía del agua: Cuestiones fundamentales. Polis. Revista Latinoamericana, 14.

Alleyne, O. (2002). Charting a future for health in the Americas: Quadrennial report of the director, 1994-1997. Charting a future for health in the Americas: quadrennial report of the director, 1994-1997.

Avina, F. (2011). Modelos de gobernabilidad democrática para el acceso al agua en América Latina. Córdoba. Tomado de http://sabersocial. virtual. avina. net/DownloadProxy. 
aspx. Blanco, A., Fretels, V., Boruchowicz, C., Herrera, K., Medellín, N., Muñoz, A., Azevedo, V., y Bouillon, C. (2012). Un espacio para el desarrollo: Los mercados de vivienda en América Latina y el Caribe.

Cabrera, H., Garcés, M., Paredes, P. (2012). Proyecto de desarrollo de capacidades para el uso seguro de aguas servidas en agricultura. Producción de Aguas Servidas, Tratamiento y Uso en el Ecuador. Pp 45-49.

Constitucional, T. (2008). Constitución de la República del Ecuador. Quito-Ecuador: Registro Oficial, 449, Pp. 20-10.

Córdova, L. A., Guerrero, L. (2015). La evacuación de las aguas servidas y su incidencia en la salubridad de los habitantes del barrio La Merced de la parroquia Juan Benigno Vela del cantón Ambato. Universidad Técnica de Ambato. Facultad de Ingeniería Civil y Mecánica

ENEMDU, INEC. (2016). Indicadores ODS de saneamiento, agua e higiene en Ecuador.

Fajardo, J. R. (2010). Desarrollo de una propuesta de gerenciamiento del recurso hídrico en la cuenca del Jubones.

Fundación IPADE. (2011). Guía de herramientas para la integración del enfoque de género en proyectos de agua y saneamiento. http://www.siagua.org/sites/default/files/documentos/docum entos/guia-genero-agua-guatemala.pdf

García, D., y Solis, H. (2011). Yakukamay. Alianza público-comunitaria: Un modelo de gestión del agua. La experiencia desde el CENAGRAP. Cenagrap/Protos-Cedir, Cañar, Ecuador.

Hantke, M. (2011). Avances legislativos en gestión sostenible y descentralizada del agua en América Latina.

Klink, F. A. (2018). Hacia una nueva economía del agua: Cuestiones fundamentales. Polis Revista Latinoamericana, 14.

Larraín, S. (2006). El agua en Chile: Entre los derechos humanos y las reglas del mercado. Polis. Revista Latinoamericana, 14.

Medina, G. (2013). La gestión comunitaria del agua mediante la alianza público-comunitaria.

Pardo, C. F. (2004). Agua y desarrollo humano. Ars Medica, 1, 12-30.

Paz, T. (2013). CAPS: Cuando la comunidad trabaja por el acceso al agua potable. https://aidaamericas.org/es/blog/caps-cuando-la-comunidad-trabajapor-el-acceso-al-agua-potable

Registro Oficial Suplemento 305. (2014). Ley organica de recursos hidricos usos y aprovechamiento del agua.
Senplades. (2014). Agua potable y alcantarillado para erradicar la pobreza en el Ecuador. https://www.planificacion.gob.ec/wpcontent/uploads/downloads/2014/09/FOLLETO-AguaSENPLADES.pdf

Serrano, F. (2008). RESERVA DE BIOSFERA PODOCARPUS - EL CÓNDOR: REFLEXIONES DEL PRIMER AÑO DE GESTIÓN. https://www.portalces.org/sites/default/files/utpl-jornadasnacionales-biologia-2008-serranofelipe.pdf

Umaña, E. M. (2016). Estrategia organizativa para el fortalecimiento a la gestión de los Comités de Agua Potable y Saneamiento en comunidades rurales del municipio de Estelí. Universidad Nacional Autónoma de Nicaragua, Managua.

Victoria, M. A. (2018). Gobernabilidad y gobernanza del agua como bien común de la naturaleza. CAMPO JURÍDICO, 6(1), 1-20. 